\title{
Ovarian Yolk Sac Tumor in a Postmenopausal Patient with Malignant Müllerian Mixed Tumor: A Case Report and Systematic Review of the Literature
}

\author{
Shuqian He \\ Shandong Qianfoshan Hospital \\ ping zhou \\ Shandong Qianfoshan Hospital \\ Yuxia Cheng \\ Shandong Qianfoshan Hospital \\ Jing Cui \\ Shandong Qianfoshan Hospital \\ Huangpeng Qi \\ Shandong Qianfoshan Hospital \\ Qing Sun ( $\nabla$ sun18653130364@163.com) \\ Shandong Qianfoshan Hospital https://orcid.org/0000-0003-3375-3597
}

\section{Case report}

Keywords: Ovary, yolk sac tumor, malignant Müllerian mixed tumor, postmenopause, prognosis.

Posted Date: November 16th, 2021

DOI: https://doi.org/10.21203/rs.3.rs-1018714/v1

License: (a) (i) This work is licensed under a Creative Commons Attribution 4.0 International License.

Read Full License 


\section{Abstract}

Background Yolk sac tumor (YST) is a most common form of malignant ovarian germ cell tumors and typically occur in young women and adolescent. Ovarian YST occurred in postmenopausal women is an extremely rare entity which has an aggressive behavior and worse outcome. The aim of this article was to provide better understanding of clinicopathological characteristics, management, and prognosis of postmenopausal YSTs.

Case presentation A 64-year-old woman was admitted to our hepatobiliary surgery department due to a cystic and solid round mass in the lower right abdomen in December 2013. laboratory tests showed afetoprotein (AFP), carcinoembryonic antigen (CEA), and cancer antigen 19-9 (CA19-9) to be within normal limits, but serum cancer antigen (CA-125) was elevated. Exploratory laparotomy was performed including tumorectomy, right oophorectomy, partial omentectomy. Postoperatively, pathologic findings showed that the morphologic features and immunohistochemical results were consistent with ovarian YST with malignant Müllerian mixed tumor (MMMT). The patient was admitted again for recurrence of the tumor and metastasis to bilateral supraclavicular lymph nodes in August 2018. The patient underwent total abdominal hysterectomy (TAH), left salpingo-oophorectomy, right salpingectomy, pelvic lymphadenectomy, and partial peritonectomy. The patient received 6 cycles of adjuvant chemotherapy with BEP (bleomycin, etoposide, and cisplatin) postoperatively, and died of disease in August 2019.

Conclusion Here, we presented a case of postmenopausal YST with MMMT. Meanwhile, a systematic literature review was conducted in the PubMed database, including case reports and case series. We summarized the clinical characteristics, treatments, and prognosis of all cases. Overall survival was calculated with Kaplan-Meier curves. Univariate analysis was performed with the log-rank test. A Cox model was constructed to determine independent predictors of mortality. As the morphological and immunophenotypical features of postmenopausal YSTs were always overlapped with somatic epithelial carcinomas, pathologists should have a high index of suspicion to diagnose ovarian YSTs in older women. Optimum chemotherapy is essential in improving prognosis of postmenopausal YSTs.

\section{Background}

Ovarian germ cell tumors (OGCTs) make up approximately $2 \%-5 \%$ of all ovarian malignancies [1]. YSTs are the second most common subtype of OGCTs and account for $14-20 \%$ of all malignant OGCTs [2]. Ovarian YSTs are encountered most frequently in the second and third decades of life and are extremely rare in postmenopausal women. YSTs occurred in postmenopausal females, either pure or mixed with somatic-type malignancies, are characterized by distinct clinicopathological characteristics and biological behavior, which can lead to diagnostic uncertainty and delayed or suboptimal treatment [3]. To the best of our knowledge, 58 postmenopausal cases of ovarian YST have been recorded since 1976. There is limited knowledge on the diagnosis, treatment, and outcome of postmenopausal YSTs. Herein, we presented a case of ovarian YST with MMMT occurred in a postmenopausal woman, meanwhile, we conducted a systematic review of 58 cases of postmenopausal YSTs, hoping to provide more meaningful 
knowledge to highlight the unique clinicopathologic features of this rare entity, identifying a significant predictor of mortality by using univariate and multivariate analysis. We believe the data summarized here will help inspire the best treatment strategies to improve the prognosis of postmenopausal YSTs in the future.

\section{Case Presentation}

In December 2013, a 64-year-old woman presented with a one-month history of lower right abdominal tenderness, and an abdominal mass was palpable on examination. The patient suffered from abdominal distension, anorexia, and constipation. The ultrasonography test detected a lower right abdominal hyperechoic mass measuring $12.2 \times 7.2 \mathrm{~cm}$. A computed tomographic scan of the abdomen demonstrated a $10 \times 8 \mathrm{~cm}$ mixed cystic and solid round mass combined with hemorrhage in the lower right abdomen, which compressed the adjacent intestine. There were no obvious signs of obstruction in the adjacent intestine. The liver, pancreas, spleen, and kidneys were otherwise normal. There were no enlarged lymph nodes in the abdominal cavity or retroperitoneum. Preoperatively, laboratory tests AFP, CEA, and CA19-9 to be within normal limits. CA-125 was elevated to $65.10 \mathrm{U} / \mathrm{ml}$ (normal, 0-35 U/ml). The tumor needs to be surgically removed because of compressing the adjacent bowel.

Three days after admission, exploratory laparotomy was performed including tumorectomy, right oophorectomy, partial omentectomy. Intraoperatively, the tumor closely adhered to the hepatic flexure of colon, ascending colon, and lower right abdominal wall. A $0.5 \times 0.5-\mathrm{cm}$ nodule could be explored in the parietal peritoneum of the lower abdominal wall. The histological report confirmed the diagnosis of ovarian YST with MMMT. Because ovarian YST with MMMT in postmenopausal women was rare, systemic treatment guidelines were unavailable. This patient received chemotherapy with calcium folinate, ftorafur, and lobaplatin. After completing one course of chemotherapy, the patient refused treatment with another course because of her poor physical condition.

In August 2018, the patient was admitted again for recurrence of the tumor and metastasis to bilateral supraclavicular lymph nodes. A computed tomographic scan of the abdomen and pelvis showed ascites and a large mass in the pelvis which closely adhered to the posterior wall of the uterus and right adnexa. Additionally, the lymph nodes could be detected in the pelvis, retroperitoneum, and bilateral groin. The serum AFP $(1.44 \mu \mathrm{g} / \mathrm{L})$, CEA $(0.82 \mu \mathrm{g} / \mathrm{L}), \mathrm{CA} 19-9(10.82 \mathrm{U} / \mathrm{ml})$ were measured Pre-operatively and all were in normal limits, but the serum CA-125 elevated to $471.1 \mathrm{U} / \mathrm{ml}$. The patient underwent TAH, left salpingooophorectomy, right salpingectomy, pelvic lymphadenectomy, and partial peritonectomy. Intraoperatively, a huge mass occupied pelvic cavity, and closely adherent to the intestine, part of the pelvic wall, and sigmoid colon. There were multiple metastatic nodules scattered on the posterior uterine wall, anterior rectal wall, and retroperitoneal area of the bladder and uterus. The FIGO stage was IV. The histological findings were consistent with those detected in the first presentation, furthermore, the tumor involved the posterior wall of the uterus and left ovary. Postoperatively, the patient received 6 cycles of adjuvant chemotherapy with BEP. After two months, the patient presented again with a recurrence of the pelvic 
tumor. A computed tomographic scan showed bilateral lung, hepatic, mediastinal, and inguinal lymph node metastasis. The patient refused further treatment and died of disease in August 2019.

\section{Pathologic Findings}

Gross pathologic examination in the first presentation revealed a cystic and solid mass that was $15 \times 10 \times 5 \mathrm{~cm}$. Part of the tumor was well encapsulated, and the cut surface was gray-yellow with some areas of cystic degeneration. Gross inspection in the second presentation revealed a hugely cystic and solid mass, measuring $19 \times 18 \times 8 \mathrm{~cm}$, and a portion of the capsule was rough. Some areas of the tumor closely adhered to the posterior uterine wall. The volume of the left adnexa was unremarkable, but some areas of the ovary were ruptured. The uterine cavity and endocervical canal were unremarkable.

Histopathological findings: The mass displayed some histologic patterns including myxomatous, solid (Fig. 1A), reticular, microcystic (Fig. 1B), glomerulus-like, gland-like, and sarcomatous formations which differed considerably from each other. Both components were distinct but typically intermingled with one another. In the reticular and myxomatous patterns, some cystic architectures are scattered in the stroma which lined by flat or pleomorphic cells with large hyperchromatic or vesicular nuclei that showed brisk mitotic activity. We can see the foci of the cystic pattern with a prominent hobnail cell appearance (Fig. 1C). Some of stromal cells could be identified by sarcomatous pattern which comprised of spindle and globoid pleomorphic cells with abundant eosinophilic cytoplasm or brisk mitotic activity (Fig. 1D). Additionally, a few chondroid components with malignant cytologic features were observed around the gland-like structure. Some of tumor cells showed epithelial-like polygonal cells with abundant clear cytoplasm. The round, brightly eosinophilic, intracelluar and extracellular PAS-positive globules were conspicuous. Immunohistochemical staining showed diffuse and strong positivity for SALL4 in reticular and some of the solid patterns (Fig. 2A), also glypican-3 was weakly positive in some of the glomeruluslike structures, while CD30, calretinin, HCG, CD117, OCT3/4, a-inhibin were negative. The strongly positivity for AFP was identified in the eosinophilic globules both inside and outside of the tumor cells (Fig. 2B). The strong positivity for Cytokeratin 7 (CK7), PAX8, EMA (Fig. 2C), and weak positivity for

Napsin A in gland-like and foci of cystic pattern with a prominent hobnail cell appearance, but WT-1, p53, and ER were negative. Desmin and vimentin were diffusely positive in sarcomatous components (Fig. 2D). The morphologic features and immunohistochemical results were consistent with ovarian YST with MMMT which was composed of an admixture of histologically malignant epithelial, mesenchymal and germ cell tumor components.

\section{Methods}

The systematic literature review included all cases of ovarian YSTs in postmenopausal women reported in the English literature from January 1976 to December 2019. A PubMed search was carried out for articles using combinations of the following keywords: ovarian, yolk sac tumor, endodermal sinus tumor, malignant ovarian germ cell tumor, postmenopause, epithelial ovarian cancer, malignant Müllerian mixed 
tumor, and carcinosarcoma. An additional literature search was also performed based on the references of the selected manuscripts. Overall survival (OS) was defined as the duration from tumor diagnosis to the date of death or last follow-up. Kaplan-Meier curves were generated to determine median and 1-year survival rates, and univariate analysis between different groups was performed using the log-rank test. Patients who were alive at the last follow-up were censored. In addition, a Cox hazard regression analysis was performed to identify factors independently associated with overall mortality. Variables statistically significantly associated with OS by univariate analysis were entered in the multivariate model. Survival analysis was restricted to cases diagnosed in 2018 and earlier. The frequency of distribution of categorical variables was compared with the chi-square test or Fisher's exact test. All statistical analyses were performed with the SPSS v.18 statistical package, and the alpha level of statistical significance was set at 0.05 .

\section{Discussion}

Ovarian YST is encountered most frequently in the second and third decades of life, followed by the first and fourth decades, and is extremely rare in postmenopausal women. Only 58 cases of ovarian YST among postmenopausal females have been recorded in the English literature during the past 45 years based on a MEDLINE database search [3-32]. We retrospectively reviewed the clinicopathological characteristics of all the published literatures of postmenopausal YSTs in Table 1. Many studies have postulated that postmenopausal YSTs have distinct molecular pathways that are different from those occurred in younger patients, leading to different clinicopathologic features and prognosis. 
Table 1

The clinicopathologic characteristics of 58 cases of ovarian yolk sac tumors in postmenopausal women.

\section{Characteristic}

Age,years

Median $=60$

Range $=50-86$

Stage

口

口

口

$\square$

N/A

Histological subtype

Pure YST

YST+MMMT

3

YST+EC/IT

3

YST+NET

2

2

28

49.1

elevated

5

normal

$\mathrm{N} / \mathrm{A}$

24

8.8

41.4

Preoperatively Serum CA125

elevated

17

29.8

normal

$\mathrm{N} / \mathrm{A}$

6

10.5

34

Site of tumor

YST: Yolk sac tumor; MMMT: malignant Müllerian mixed tumor; EC: embryonal carcinoma; IT: immature teratoma; NET: neuroendocrine tumor; DFS: disease-free survival; OS: overall survival; DOD: died of disease; BEP: bleomycin, etoposide, cisplatin; TC: paclitaxel, carboplatin; N/A: not avaiable. 


\begin{tabular}{|c|c|c|}
\hline Characteristic & Number of patients & $\%$ \\
\hline left ovary & 18 & 31.6 \\
\hline right ovary & 29 & 50.9 \\
\hline Bilateral ovary & 6 & 10.5 \\
\hline Uterus & 1 & 1.8 \\
\hline $\mathrm{N} / \mathrm{A}$ & 3 & 5.3 \\
\hline \multicolumn{3}{|c|}{ Tumor size $($ median $=20 \mathrm{~cm})$} \\
\hline$<20 \mathrm{~cm}$ & 21 & 36.2 \\
\hline$\geqq 20 \mathrm{~cm}$ & 7 & 12.1 \\
\hline $\mathrm{N} / \mathrm{A}$ & 30 & 51.7 \\
\hline \multicolumn{3}{|c|}{ Adjuvant Chemotheraphy } \\
\hline Yes & 33 & 56.9 \\
\hline BEP & 10 & 17.2 \\
\hline TC & 4 & 6.9 \\
\hline BEP and TC & 4 & 6.9 \\
\hline Others & 15 & 25.9 \\
\hline No & 6 & 10.3 \\
\hline $\mathrm{N} / \mathrm{A}$ & 19 & 32.8 \\
\hline \multicolumn{3}{|c|}{ Outcome(DFS median=24 months; OS median=10 months) } \\
\hline DFS $<24$ months & 10 & 17.2 \\
\hline $\mathrm{DFS} \geqq 24$ months & 6 & 10.3 \\
\hline DOD $<10$ months & 12 & 20.7 \\
\hline $\mathrm{DOD} \geqq 10$ months & 15 & 25.7 \\
\hline N/A & 15 & 25.9 \\
\hline
\end{tabular}

Postmenopausal YSTs occur most often in association with a somatic-type malignancy. The most commonly associated somatic neoplasm is endometrioid carcinoma. secondly, other associated tumors were high-grade serous carcinoma, clear cell carcinoma, mucinous tumors, and MMMT. Up to now, the pathogenesis of postmenopausal YSTs has not been well understood. There are four theories to 
recommend: the teratoma theory, retrodifferentiation, the collision theory, and the neometaplasia theory $[6,16,18,30]$. The theory that YSTs originate from germ cells is highly unlikely at an older age, as germ cells are not identifiable histologically in the ovaries of the postmenopausal female. Nogales [11]postulated that both components of postmenopausal YSTs arise from a common, non-germ-cell precursor. Neometaplasia, known as aberrant differentiation, refers to the germ cell component that arises through a process of transformation of the epithelial precursor neoplasm [6, 30]. The most frequently reported epithelial precursor neoplasm is endometrioid carcinoma, which sometimes develops from endometriosis or endometriotic cysts [33]. This is in line with the hypothesis that endometriosis or endometriotic cysts may be a risk factor for YST in postmenopausal women [24]. However, the molecular events involved in this transformation have not yet been elucidated. Many reports consider that the germ cells are developed from teratoma to be unlikely, as most of the tumors lack the histologic diversity and presence of immature embryonic tissue seen in immature teratoma or the more mature fetal or adult tissue seen in mature cystic teratoma associated with carcinoma. Herein, we report a new case of ovarian YST in a 64-year-old woman. We identified diverse differentiation of the tumor based on morphologic features and the immunohistochemical profile, including YST, Müllerian epithelial components, heterologous elements including chondrosarcoma and rhabdomyosarcoma, and the intestinal variant. A variety of tumor components are also considered to be derived from the somatic component by a process of "neometaplasia" or "retrodifferentiation".

In fetal development, AFP is produced by the yolk sac, liver, and upper gastrointestinal tract. It has been demonstrated that germ cell tumors in patients with elevated serum AFP are either composed entirely of or contain yolk sac tumor elements [34]. Some studies have shown that serum AFP always elevated preoperatively in YSTs. In our study, the serum AFP was measured pre-operatively only in 33 cases, in which elevated AFP was in 28 cases, and normal in 5 cases. Boussios suggested that in older women with an ovarian mass and an elevated serum AFP level, a diagnosis of ovarian YST should be suspected [3]. Meanwhile, we found that the serum AFP level was not different between pure YSTs and mixed YSTs in postmenopausal women ( $P>0.05)$. There were 2 cases of ovarian YST mixed with a MMMT reported in the previous English literature. The serum AFP level was elevated slightly to $23 \mu \mathrm{g} / \mathrm{L}$ (normal $0-20 \mu \mathrm{g} / \mathrm{L}$ ) in one case, and corresponding data in another case were not available. It is interesting that the serum AFP of our case reported here did not elevate pre-operatively or post-operatively. It should be noted that the serum AFP determination is useful diagnostically and in monitoring therapy, but a normal result may not always indicate the absence of active disease, especially in postmenopausal females. CA-125 has been found to be a valuable tumor marker in adenocarcinoma patients. The reported cases of ovarian YSTs had elevated serum CA-125 in 17 patients, and most of them were mixed YSTs which were associated with epithelial cancer. The serum CA-125 elevated to $65.10 \mathrm{U} / \mathrm{ml}$ (normal, 0-35 U/ml) in our case. It should be borne in mind that the diagnosis of ovarian YST even if the serum CA-125 elevated but AFP was negative result in older women.

The morphological and immunophenotypical features of such tumors markedly overlapped with epithelial neoplasms such as clear cell carcinoma, endometrioid carcinoma, serous carcinoma and malignant Müllerian mixed tumor which can make diagnosis difficult. An immunohistochemical panel, 
including CK7, EMA, PAX-8, WT-1, estrogen receptor (ER), progesterone receptor (PR), HNF-1, and Napsin $A$, should be applied for identifying the diverse tumor components. It is generally accepted that the positive immunostains for AFP, SALL4, and glypican-3 are valuable markers in identifying YSTs components histologically [35]. SALL4, a zinc finger transcription factor, is a specific marker for germ cell tumors, but in addition to YSTs, it is positive in dysgerminoma and embryonal carcinoma, among other tumors. Although, SALL4 appears to be the more sensitive marker for distinguishing YST from other epithelial tumors [21], the aberrant staining patterns were always likely to occur and the positivity of SALL4 was found in more than $20 \%$ of ovarian serous carcinomas [36]. AFP is more specific for YSTs, and can be identified not only in the tumor cells but also in the eosinophilic globules present both inside and outside the tumor cells, but the immunostains of AFP always was weak or negative. Glypican-3, an oncofetal protein expressed in the fetal liver and malignant tumors of hepatocytic lineage, is more sensitive than AFP but not as specific [37]. Positive glypican-3 appears variable in clear cell carcinoma, and when positive, it does not reliably distinguish it from YSTs [38]. There are significant overlap in the immunophenotype between postmenopausal YSTs and epithelial neoplasms. CK7 and EMA were often positive in glandular YSTs, sometimes diffusely, which may reflect the probable derivation of the YST components from the somatic epithelial neoplasms [30]. Other germ cell tumors, such as dysgerminoma, express OCT3/4, CD117 and D2-40, in addition to SALL4, which can be differentiated from YSTs. Embryonal carcinoma is usually positive for OCT-3/4 and CD30 and negative for SALL4, AFP and glypican-3, while YST typically displays the opposite pattern. Positivea-inhibin and calretinin can be used to distinguish adult granulosa cell tumors from YSTs. The morphological and immunohistochemical features of our case were favour of a diagnosis of ovarian YST associated with MMMT. For the ovarian tumors occurred in older women with complex morphologically components, a set of immunophenotypes should be used for diagnosis and differential diagnosis.

Due to the malignancy of postmenopausal YSTs, the prognosis is poor, and the three-year survival rate is $13 \%$ [28]. Some studies have demonstrated that the tumor stage [39], serum AFP decline rate[40], residual tumor [41], volume of ascites [40] were prognosis-related factors in ovarian YSTs. Since the introduction of several combination chemotherapy regimens, including CBC (cisplatin-based chemotherapy), VBP (vinblastine, bleomycin, cisplatin)., PVP (cisplatin, vinblastine, peplomycin), TC (paclitaxel, carboplatin) and BEP, et al, the prognosis of ovarian YSTs has dramatically improved. For women with an ovarian YST treated with the BEP regimen, the 5-year survival rate approaches $94 \%$, and even in patients with advanced-stage disease [39], consequently, the BEP regimen has been taken for the standard of care for ovarian YSTs. Platinum-based adjuvant chemotherapy aiming to treat both epithelial ovarian neoplasms and germ cell tumors, which are components that are identifiable or postulated to be a part of the tumor at one time, has also been used. It was reported that postmenopausal YSTs may be less sensitive to chemotherapy than those that arise de novo [18], as the tumor component arises by transformation of an epithelial ovarian neoplasm. To date, there wasn't a report on the analysis of prognosis-related factors for postmenopausal YSTs, moreover, there was no effective chemotherapy regimens recommended due to the rarity. Herein, we showed that advanced-stage disease (HR=5.367, $P=0.0205)$ and different adjuvant chemotherapy regimen $(\mathrm{HR}=3.992, P=0.0004)$ were associated with poor overall survival by using 
univariate and multivariate analysis, and the selection of adjuvant chemotherapy regimen (HR=1.091, $P=0.003$ ) was a favorable independent prognostic factor for postmenopausal YSTs, whether the somatictype malignancies were mixed with or not. Age, serum AFP, serum CA125, tumor size and tumor location were not associated with overall survival $(P>0.05)$. What calls for special attention is that the first-line adjuvant chemotheraphy regimen with BEP or TC or the combination of BEP and TC may be quite effective against postmenopausal YSTs, but more clinical data will need to be accumulated in the future.

\section{Conclusion}

Herein, we report a case of postmenopausal YST associated with MMMT and retrospectively analyze 58 cases of postmenopausal YSTs reported in the English literature. The ovarian YST was an rare entity occurred in menopausal women and most of them were associated with a somatic-type neoplasm, which increased diagnostic difficulty and confusion, and the systemic treatment guidelines were unavailable. To date, this is first study on the analysis of prognosis-related factors for ovarian YSTs occured in postmenopausal women using univariate and multivariate analysis. It is worth pointing out that the adjuvant chemotheraphy with BEP or TC or the combination of BEP plus TC regimen can be recommended as the first-line choices for postmenopausal YSTs. It is hoped that a better understanding of the unique clinicopathological characteristics of ovarian YSTs in the postmenopausal females can be beneficial to explore better treatment methods and improve the prognosis of this rare entity.

\section{Abbreviations}

OGCTS

Ovarian germ cell tumors

YST

Yolk sac tumor

MMMT

Malignant Müllerian mixed tumor

AFP

a-fetoprotein

CEA

Carcinoembryonic antigen

CA19-9

Cancer antigen 19-9

CA- 125

serum cancer antigen

$\mathrm{TAH}$

abdominal hysterectomy

BEP

bleomycin, etoposide, cisplatin 
ER

Estrogen receptor

PR

Progesterone receptor

CBC

Cisplatin-based chemotherapy

VBP

vinblastine, bleomycin, cisplatin

PVP

cisplatin, vinblastine, peplomycin

TC

paclitaxel, carboplatin

EC

Embryonal carcinoma

IT

Immature teratoma

NET

Neuroendocrine tumor

DFS

Disease-free survival

OS

overall survival, DOD:Died of disease

$\mathrm{N} / \mathrm{A}$

Not avaiable.

\section{Declarations}

\section{Acknowledgements}

None

\section{Funding}

This study was supported by Shandong Province Key Research and Development Program (No.2017CXGC1201, 2019JZZY010108). Medical and healthy technology development Foundation, Health commission of Shandong Province (No.2015WS0244, 2015WS0249). National Nature Science Foundation of China (81301789). Natural Science Foundation of Shandong Province (ZR2011HQ013).

\section{Availability of data and materials}

The datasets used and analysed during the current study are availiable from the corresponding author on reasonable request. 


\section{Author's contributions}

SQH conceived and designed the study and edited the final manuscript. PZ was responsible for the literature review and the data collection. JC contributed to the statistical analyses. YXC performed the immunohistochemical staining. HPQ prepared the electronic artwork data analysis. QS was responsible for manuscript revising. All authors have read and approved the manuscript. All authors contributed to the article and approved the submitted version.

\section{Ethical approval and consent to participate}

The study was approved by the ethics committee of The First Affiliated Hospital of Shandong First Medical University.

\section{Consent for publication}

Informed consent was obtained from the patient.

\section{Competing interests}

The authors declare that they have no competing interest.

\section{Authors details}

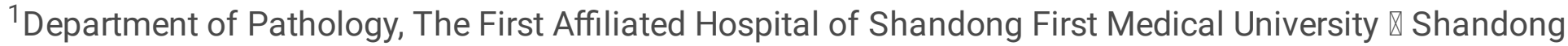
Provincial Qianfoshan Hospital, Shandong Medicine and Health Key Laboratory of Clinical Pathology, Shandong Lung Cancer Institute, Shandong Institute of Nephrology, Jinan, Shandong, P.R. China

\section{References}

1. Low JJ, Ilancheran A, Ng JS. Malignant ovarian germ-cell tumours. Best Pract Res Clin Obstet Gynaecol. 2012;26(3):347-55.

2. Tewari K, Cappuccini F, Disaia PJ, Berman ML, Manetta A, Kohler MF. Malignant germ cell tumors of the ovary. Obstet Gynecol. 2000;95(1):128-33.

3. Boussios S, Attygalle A, Hazell S, Moschetta M, Mclachlan J, Okines A, et al. Malignant Ovarian Germ Cell Tumors in Postmenopausal Patients: The Royal Marsden Experience and Literature Review. Anticancer Res. 2015;35(12):6713.

4. Brown JR, Green JD. Yolk sac carcinoma. South Med J. 1976;69(6):728-31.

5. Ferracini R, Gardini G, Lanzanova G, Lorenzini P. Endodermal sinus tumor in a 63 year old female. Pathologica. 1979;71(1016):885.

6. Rutgers JL, Young RH, Scully RE. Ovarian yolk sac tumor arising from an endometrioid carcinoma. Hum Pathol.1987;18(12):1296-9. 
7. Mazur MT, Talbot WJ, Talerman A. Endodermal sinus tumor and mucinous cystadenofibroma of the ovary. Occurrence in an 82-year-old woman. Cancer-Am Cancer Soc. 1988;62(9):2011-5.

8. Kinoshita K. A 62-year-old woman with endodermal sinus tumor of the ovary. Am J Obstet Gynecol. 1990; 162(3):760.

9. Pliskow S. Endodermal sinus tumor of the ovary: review of 10 cases. South Med J. 1993; 86(2):1879.

10. Kammerer-Doak D, Baurick K, Black W, Barbo DM, Smith HO. Endodermal Sinus Tumor and Embryonal Carcinoma of the Ovary in a 53-Year-Old Woman. Gynecol Oncol.1996; 63(1):133-7.

11. Nogales FF, Bergeron C, Carvia RE, Alvaro T, Fulwood HR. Ovarian endometrioid tumors with yolk sac tumor component, an unusual form of ovarian neoplasm. Analysis of six cases. Am J Surg Pathol. 1996; 20(9):1056-66.

12. Takizawa K, Kawana T, Kakinoki S, Saito R, Takeda Y. Case report of a 69-year-old woman with double cancers: Primary yolk sac tumor of the right ovary and primary serous surface papillary carcinoma of the peritoneum. Int J Clin Oncol. 1996;1(3):190-4.

13. Horiuchi A, Osada R, Nakayama K, Toki T, Nikaido T, Fujii S. Ovarian yolk sac tumor with endometrioid carcinoma arising from endometriosis in a postmenopausal woman, with special reference to expression of alpha-fetoprotein, sex steroid receptors, and p53. Gynecol Oncol. 1998; 70(2):295-9.

14. Arai T, Kitayama Y, Koda K. Ovarian Mucinous Cystadenocarcinoma with Yolk- Sac Tumor-in a 71Year-Old Woman. Int J Gynecol Pathol. 1999;18(3):277-80.

15. Oh C, Kendler A, Hernandez E. Ovarian Endodermal Sinus Tumor in a Postmenopausal Woman. Gynecol Oncol. 2001; 82(2):392-4.

16. Seiryu K, Yoshiharu O, Osamu M, Susumu O, Mariko S, Norihiro M, et al. A case of ovarian endometrioid adenocarcinoma with yolk sac tumor component in a postmenopausal woman. Apmis. 2002;110(6):508-14.

17. Filiz G, Ozuysal S, Bilgin T. Ovarian endodermal sinus tumor in a 76-year-old woman. J Obstet Gynaecol Res. 2003;29(5):309-11.

18. Lopez JM, Malpica A, Deavers MT, Ayala AG. Ovarian yolk sac tumor associated with endometrioid carcinoma and mucinous cystadenoma of the ovary. Ann Diagn Pathol. 2003; 7(5):300-05.

19. Garcia-Galvis OF, Cabrera-Ozoria C, Fernandez JA, Stolnicu S, Nogales FF. Malignant Mullerian mixed tumor of the ovary associated with yolk sac tumor, neuroepithelial and trophoblastic differentiation (teratoid carcinosarcoma). Int J Gynecol Pathol. 2008; 27(4):515-20.

20. Abe A, Furumoto H, Yoshida K, Nishimura M, Irahara M, Kudo E, et al. A case of ovarian endometrioid adenocarcinoma with a yolk sac tumor component. Int J Gynecol Cancer. 2008; 18(1):168-72.

21. Roth LM, Talerman A, Levy T, Sukmanov O, Czernobilsky B. Ovarian Yolk Sac Tumors in Older Women Arising From Epithelial Ovarian Tumors or With No Detectable Epithelial Component. Int J Gynecol Pathol. 2011;30(5):442-51. 
22. Lange S, Livasy C, Tait DL. Endodermal sinus tumor of the ovary in an 86 year old woman. Gynecologic Oncology Case Reports. 2012;2(2):65-6.

23. Varia M, Mccluggage WG, Oommen R. High grade serous carcinoma of the ovary with a yolk sac tumour component in a postmenopausal woman: report of an extremely rare phenomenon. J Clin Pathol. 2012;65(9):853-4.

24. Koi C, Kurita T, Kagami S, Matsuyaya A, Hachisuga T. A case of ovarian yolk sac tumor associated with endometrioid adenocarcinoma. Gynecologic Oncology Case Reports. 2014; 9:11-4.

25. Roma AA, Przybycin CG. Yolk Sac Tumor in Postmenopausal Patients: Pure or Associated With Adenocarcinoma, a Rare Phenomenon. Int J Gynecol Pathol. 2014;33(5):477-82.

26. Chen Q, Chen X. Bilateral ovarian mixed epithelial adenocarcinoma in a postmenopausal woman with unilateral ovarian yolk sac tumor component. Int J Clin Exp Patho. 2014;7(11):8259.

27. Parker VL, Sanderson P, Naik V, Quincey C, Farag K. Post-menopausal presentation of yolk sac germ cell tumour. Gynecologic Oncology Reports. 2015; 11:16-9.

28. Wang $X$, He J, Li Y. Ovarian yolk sac tumor in postmenopausal females: a report of five cases and a literature review. Eur J Gynaecol Oncol. 2016;37(3):374-9.

29. Mccarthy WA, Masand RP. Ovarian Yolk Sac Tumor With High-Grade Serous Carcinoma in a 62-YearOld Woman. Int J Surg Pathol. 2016;24(4):360-5.

30. Mcnamee T, Damato S, Mccluggage WG. Yolk sac tumours of the female genital tract in older adults derive commonly from somatic epithelial neoplasms: somatically derived yolk sac tumours. Histopathology. 2016;69(5):739-51.

31. Taranto P, Carvalho FM, Roithmann S, Maluf FC. Ovarian yolk sac tumor coexisting with epithelial ovarian cancer: An aggressive rare entity. Gynecol Oncol Rep. 2017;22:37-9.

32. Wang Y, Yang J, Yu M, Cao D, Zhang Y, Zong X, et al. Ovarian yolk sac tumor in postmenopausal females: A case series and a literature review. Medicine (Baltimore). 2018; 97(33):e11838.

33. Robinson KA, Menias CO, Chen L, Schiappacasse G, Shaaban AM, Caserta MP, et al. Understanding malignant transformation of endometriosis: imaging features with pathologic correlation .Abdom Radiol(NY). 2020;45(6):1762-75.

34. Norgaard-Pedersen B, Albrechtsen R, Teilum G. Serum alpha-foetoprotein as a marker for endodermal sinus tumour (yolk sac tumour) or a vitelline component of "teratocarcinoma". Acta Pathol Microbiol Scand A.1975;83(6):573-89.

35. Nogales FF, Quinonez E, Lopez-Marin L, Dulcey I, Preda O. A diagnostic immunohistochemical panel for yolk sac (primitive endodermal) tumours based on an immunohistochemical comparison with the human yolk sac. Histopathology. 2014;65(1):51-9.

36. Nogales FF, Dulcey I, Preda O. Germ cell tumors of the ovary: an update. Arch Pathol Lab Med. 2014;138(3):351-62.

37. Zynger DL, Mccallum JC, Luan C, Chou PM, Yang XJ. Glypican 3 has a higher sensitivity than alphafetoprotein for testicular and ovarian yolk sac tumour: immunohistochemical investigation with 
analysis of histological growth patterns. Histopathology. 2010; 56(6):750-7.

38. Dällenbach P, Bonnefoi H, Pelte MF, Vlastos G. Yolk sac tumours of the ovary: an up date. European Journal of Surgical Oncology (EJSO). 2006;32(10):1063-75.

39. Solheim O, Gershenson DM, Tropé CG, Rokkones E, Sun CC, Weedon-Fekjaer H, et al. Prognostic factors in malignant ovarian germ cell tumours (The Surveillance, Epidemiology and End Results experience 1978-2010). Eur J Cancer. 2014;50(11):1942-50.

40. de La Motte RT, Pautier P, Rey A, Duvillard P, Kerbrat P, Troalen F, et al. Prognostic factors in women treated for ovarian yolk sac tumour: a retrospective analysis of 84 cases. Eur $\mathrm{J}$ Cancer. 2011;47(2):175-82.

41. Nawa A, Obata N, Kikkawa F, Kawai M, Nagasaka T, Goto S, et al. Prognostic factors of patients with yolk sac tumors of the ovary. Am J Obstet Gynecol. 2001;184(6):1182-8.

\section{Figures}
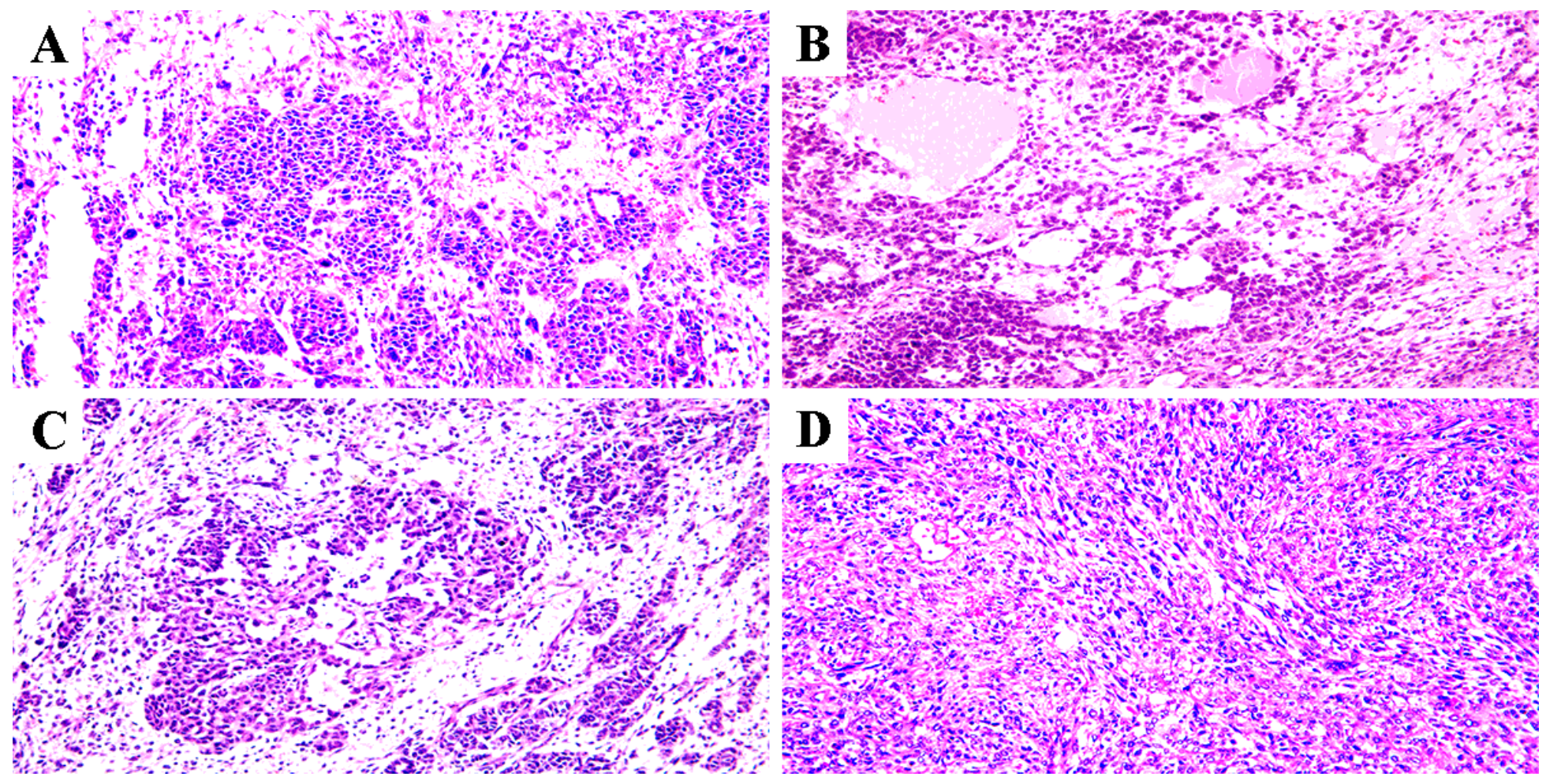

\section{Figure 1}

Title: Histopathological image of a case of YST with MMMT. Legends: $a, b$ Yolk sac tumor $(H$ and $E, \times 10)$. reticular, myxomatous, solid and microcystic pattern. c Foci of clear cell carcinoma with a prominent hobnail cell appearance $(H$ and $E, \times 10)$. $d$ The area of rhabdomyosarcoma with brisk mitotic activity $(H$ and $E, \times 10)$. 




Figure 2

Title: Immunophenotypical features of YST with MMMT. Legends: a Diffuse and strong positivity for SALL4 in YST components $(\times 20)$. b Strong positivity for AFP in eosinophilic globules inside and outside the tumor cells $(\times 20)$ (arrow). c Strong and diffuse positivity for EMA in clear cell carcinoma components $(\times 10)$. d Diffuse positivity for desmin in rhabdomyosarcoma components $(\times 10)$.
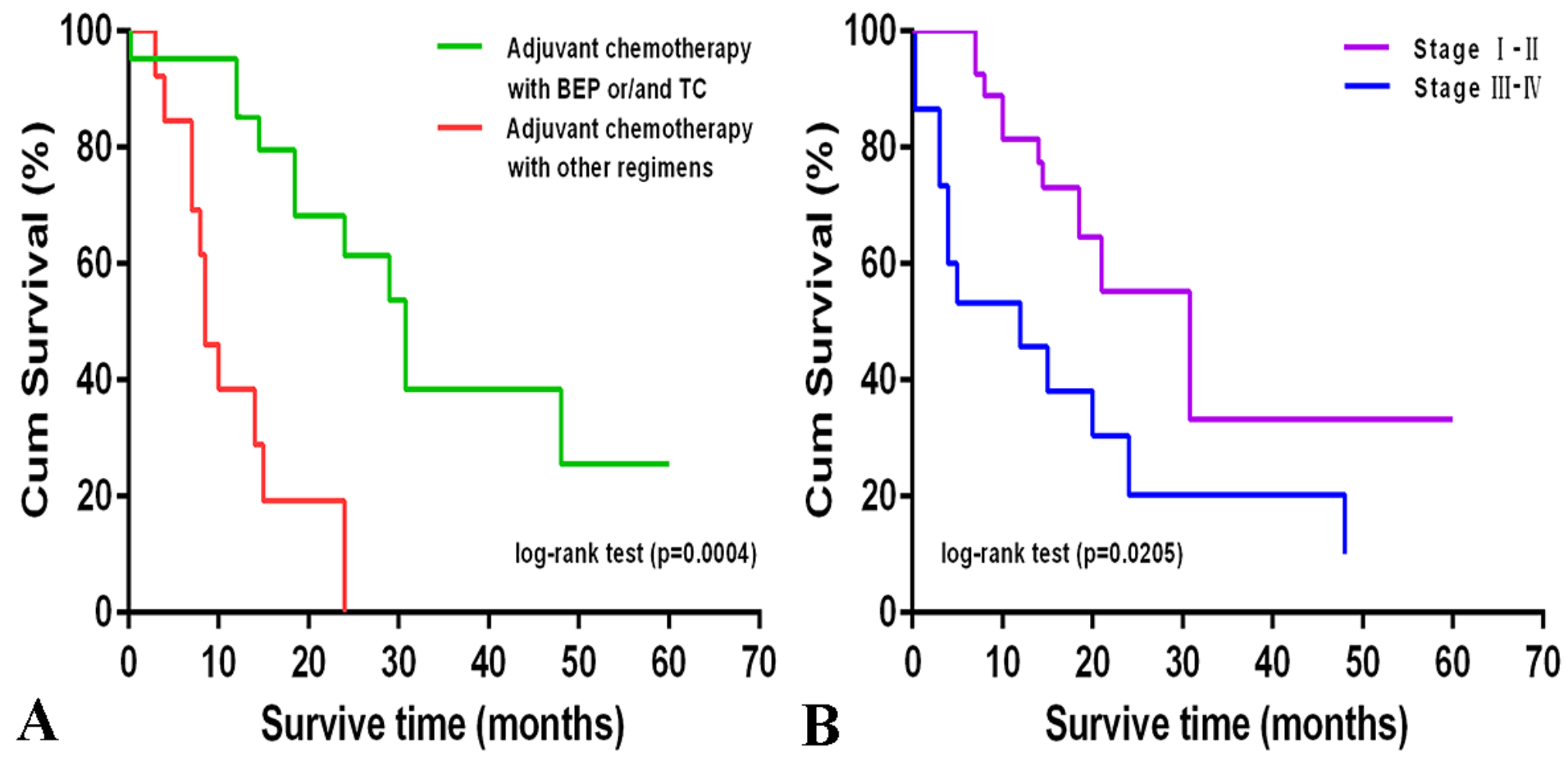

Figure 3 
Title: The analysis of prognosis-related factors in postmenopausal YSTs. Legends: a overall survival of surgically managed with postmenopausal YSTs stratified by receipt of adjuvant chemotherapy $(n=18$ for chemotherapy with BEP or TC or the combination with BEP and TC, $n=10$ for chemotherapy with other chemotherapy regimens, $\mathrm{P} \otimes 0.001$ from log-rank test). b overall survival of surgically managed with postmenopausal YSTs stratified by disease stage ( $n=30$ for stage I-II, $n=20$ for stage III-IV, Pख0.05 from log-rank test).

\section{Supplementary Files}

This is a list of supplementary files associated with this preprint. Click to download.

- supplementaryTable.docx 